Onkologe 2016 $\cdot 22: 904-907$

DOI 10.1007/s00761-016-0131-4

(C) Springer-Verlag Berlin Heidelberg 2016

CrossMark

\author{
Angelika Eggert ${ }^{1} \cdot$ Dirk Reinhardt $^{2} \cdot$ Christian Kratz $^{3}$ Udo Kontny ${ }^{4}$. \\ Dominik T. Schneider ${ }^{5} \cdot$ Heribert Jürgens ${ }^{6} \cdot$ Heinz Schmidberger $^{7}$ \\ 'Klinik für Pädiatrie mit Schwerpunkt Onkologie und Hämatologie, Charité Universitätsmedizin Berlin, \\ Berlin, Deutschland \\ ${ }^{2}$ Klinik für Kinderheilkunde III, Universitätsklinikum Essen, Essen, Deutschland \\ ${ }^{3}$ Klinik für Pädiatrische Onkologie und Hämatologie, Medizinische Hochschule Hannover, Hannover, \\ Deutschland \\ ${ }^{4}$ Klinik für Kinder- und Jugendmedizin, Universitätsklinikum Aachen, Aachen, Deutschland \\ ${ }^{5}$ Klinik für Kinder- und Jugendmedizin, Klinikum Dortmund, Dortmund, Deutschland \\ ${ }^{6}$ Klinik für Pädiatrische Onkologie und Hämatologie, Universitätsklinikum Münster, Münster, Deutschland \\ ${ }^{7}$ Unimedizin Mainz, Mainz, Deutschland
}

\title{
Pädiatrische Onkologie
}

\section{Behandlungsstrukturen und aktuelle Herausforderungen}

In Deutschland erkranken jährlich ca. 2000 Kinder und Jugendliche neu an einer Krebserkrankung. Während bei Erwachsenen die Karzinome $>90 \%$ der Neuerkrankungen ausmachen, sind diese bei Kindern mit $<1 \%$ sehr selten. Daher lassen sich Erkenntnisse aus der Erwachsenenonkologie nur zu einem geringen Teil auf die pädiatrische Onkologie übertragen. Die häufigsten Erkrankungen bei Kindern sind Leukämien (34\%), Hirntumoren (23\%), Lymphome (12\%) und Neuroblastome (8\%). Vor allem die enge interdisziplinäre Zusammenarbeit von behandelnden Ärzten, Pflegepersonal, psychosozialem Dienst und Forschern in den vergangenen Jahrzehnten hat den Kampf gegen Krebs bei Kindern zu einer Erfolgsgeschichte der Medizin gemacht, sodass in den Industrieländern heute ca. 80 \% der betroffenen Patienten überleben.

\section{Behandlungsstruktur der pädiatrischen Onkologie}

Die erfreulichen Heilungsraten in der pädiatrischen Onkologie wurden v. a. durch den Aufbau kooperativer Struk-

Angelika Eggert, Dirk Reinhardt, Christian Kratz, Udo Konty, Dominik T. Schneider und Heribert Jürgens repräsentieren die Gesellschaft für Pädiatrische Onkologie und Hämatologie (GPOH) turen für Erfassung, Diagnostik, Therapie und Nachsorge pädiatrisch-onkologischer Erkrankungen unter dem Dach der GPOH ermöglicht. Zu den wesentlichen Strukturelementen gehören:

- die Therapieoptimierungsstudien der GPOH mit den jeweiligen diagnostischen und therapeutischen Referenzzentren,

- das Deutsche Kinderkrebsregister am Institut für Medizinische Biometrie, Epidemiologie und Informatik (IMBEI) an der Johannes-Gutenberg Universität Mainz,

- das Deutsche Kindertumorregister an der Universität Schleswig Holstein,

- die GPOH-Biomaterialbanken,

- das Pädiatrische Register für Stammzelltransplantation an der Universität Frankfurt,

- die Arbeitsgemeinschaft für Pädiatrische Radioonkologie (APRO) der GPOH und der DEGRO (Deutsche Gesellschaft für Radioonkologie),

- Arbeitsgemeinschaften und Arbeitsgruppen u. a. zur Lebensqualität, psychosozialen Betreuung (PSAPOH) und Erfassung von Spätfolgen (LESS),

- aktuell 58 spezialisierte Behandlungszentren, die die erforderlichen Infrastrukturkriterien nach Vorgabe des Gemeinsamen Bundesauschuss (G-BA) erfüllen.
Die geringe Inzidenz pädiatrischer Krebserkrankungen und die Notwendigkeit einer hohen ärztlichen und pflegerischen Expertise in der Steuerung der Therapie und ihrer Nebenwirkungen haben $\mathrm{zu}$ einer regionalen Zentralisierung der Patientenversorgung in aktuell 58 spezialisierten Kliniken geführt. Eine weitere Zentralisierung ist in den nächsten Jahren durch die Einführung eines pädiatrischen Moduls im Zertifizierungsverfahren der Deutschen Krebsgesellschaft (OnkoZert) sowie durch eine zunehmende Spezialisierung großer Zentren und Forschungsverbünde auf die Durchführung molekularer Diagnostik und klinischer Phase-I/II-Studien zu erwarten.

Der G-BA hat 2006 die gesetzlichen Voraussetzungen für die Zulassung pädiatrisch-onkologischer Zentren zur Behandlung dieser Patienten definiert. Bereits bei Verdacht auf eine Krebserkrankung müssen Kinder und Jugendliche gemäß dieser Vorgabe in ein kinderonkologisches Behandlungszentrum überwiesen werden, in dem eine fachkompetente ärztliche, pflegerische und psychosoziale Versorgung nach den Infrastrukturkriterien des G-BA gewährleistet ist (www. g-ba.de/informationen/richtlinien/47).

Darüber hinaus ist auch in großen Zentren nur dann ein adäquater Therapieerfolg möglich, wenn eine enge 
Hier steht eine Anzeige.

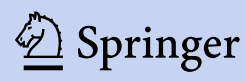


Zusammenarbeit mit Spezialisten anderer Fachrichtungen (z. B. Kinderchirurgen, pädiatrische Neurochirurgen/Radiologen/Strahlentherapeuten) erfolgt. Zudem ist die psychosoziale Versorgung seit 25 Jahren unverzichtbarer Bestandteil der pädiatrisch-onkologischen Gesamtbehandlung. Die psychosozialen Versorgungsangebote haben sich im klinischen Alltag bewährt. Bedarf und Wirksamkeit konnten in zahlreichen Studien belegt werden.

Die wichtigste Qualitätssicherungsmaßnahme stellen darüber hinaus die sog. Therapieoptimierungsstudien und Register dar, in denen deutschlandweit $>90 \%$ aller Kinder und Jugendlichen mit einer Krebserkrankung diagnostiziert und behandelt werden. Diese Studien dienen der Qualitätssicherung, Standardisierung und Optimierung von Therapieoptionen. Die weitere Verbesserung der Behandlungsergebnisse wird durch Diagnostik und Therapie nach dem aktuellen Stand des Wissens und auf der Basis der Erkenntnisse vorangegangener Studien erzielt. Es geht hierbei also nicht um die Zulassung neuer Medikamente, sondern um die kontinuierliche Verbesserung des gesamten Therapiekonzepts. Die Kinderonkologie belegt beispielhaft, wie durch die konsequente Erfassung aller Erkrankungen in einem Krebsregister und ein einheitliches, multizentrisches, evidenzbasiertes Vorgehen die Prognose einer Krebserkrankung verbessert werden kann.

\section{Generelle Konzepte in Diagnostik und Therapie}

Diagnostik und Therapie müssen konsequent nach den Vorgaben der jeweiligen Therapieoptimierungsstudie erfolgen, um die Heilungschancen der Patienten zu wahren und Nebenwirkungen zu begrenzen. Behandlungsverläufe und -ergebnisse werden detailliert dokumentiert und ausgewertet, um aus den erhobenen Daten Rückschlüsse auf die Qualität und den Erfolg des Behandlungskonzepts zu ziehen. Nachfolgende Therapiekonzepte führen diese Erkenntnisse der klinischen Forschung dann mit neuen Ergebnissen der Grundlagenforschung zusammen, um die Therapie in der jeweiligen Folgestudie weiter zu optimieren.

Prinzipiell erfolgt die Diagnostik in der pädiatrischen Onkologie nach den gleichen Prinzipien, die in der Onkologie bei Erwachsenen gelten. Die Initialdiagnostik sollte ausnahmslos in einer pädiatrisch-onkologischen Klinik erfolgen, denn eine kritische Auswahl der Untersuchungsverfahren und Interpretation ihrer Ergebnisse ist die Voraussetzung für eine korrekte Diagnose. Angesichts der oft raschen Wachstumskinetik der embryonalen Tumoren ist eine zügige Diagnostik indiziert. Generell erfolgt die onkologische Therapie bei Kindern multimodal und risikoadaptiert. Eine onkologische Primärtherapie außerhalb der etablierten Therapieoptimierungsstudien ist abzulehnen und gilt als ärztlicher Kunstfehler, da bereits geringfügige $\mathrm{Ab}$ weichungen von den aktuellen Standards die erreichbaren günstigen Ergebnisse gefährden können. In diagnostisch oder therapeutisch unklaren Situationen sind die Referenzeinrichtungen und Studienzentralen zwingend miteinzubeziehen. Da die oft beachtlichen Behandlungserfolge bislang auf dieser Basis erreicht wurden, erfolgt auch der Einsatz neuerer Zytostatika oder molekular gezielter Medikamente in der Primärtherapie kindlicher Malignome ausschließlich im Rahmen klinischer Studienprotokolle.

Da der Zeitpunkt der definitiven Operation im Rahmen eines multidisziplinären Gesamtkonzepts der Therapie entscheidend von der definitiven Diagnose abhängt und in vielen Fällen eine initiale Biopsie ausreichend ist, ist eine OPPlanung im Rahmen interdisziplinärer Tumorkonferenzen unter Federführung eines pädiatrischen Onkologen von zentraler Bedeutung. Der Operateur muss in der pädiatrischen Tumorchirurgie erfahren sein. Aufgrund der Seltenheit von Tumoren im Kindes- und Jugendalter ist deshalb nicht selten eine Verlegung in ein erfahrenes kinderchirurgisches Zentrum erforderlich. Für die meisten Tumorentitäten ist inzwischen eine komplexe molekulargenetische Untersuchung des Tumormaterials zur Risikoadaptation der Therapie erforderlich. Neben den diagnostischen Referenzzentren sind funktionierende Biomaterialbanken hierfür die Voraussetzung. In speziell ausgerüsteten Behältnissen (GPOH Tumorbox ${ }^{\circledR}$ ) werden Tumormaterial und andere Gewebe- oder Blutproben direkt aus dem $\mathrm{OP}$ an die zentrale GPOH-Biobank und die betreffenden zentralen Referenzeinrichtungen gesandt.

Die Strahlentherapie ist neben der Chemotherapie bei vielen Tumoren ein unverzichtbarer Bestandteil der multimodalen Kombinationstherapie und wird sowohl in kurativen Konzepten als auch in der Palliativtherapie eingesetzt. Auch die Radiotherapie von Kindern sollte nur in Kliniken erfolgen, die über entsprechende Erfahrung auf diesem Gebiet verfügen. Indikationsstellungen und die Festlegung der zu bestrahlenden Region, der Dosierungen und Fraktionierungen sowie der Bestrahlungsmodalität werden für jede Tumorart in der Arbeitsgemeinschaft für Pädiatrische Radioonkologie (APRO) beraten. Sie werden für die einzelnen Therapieprotokolle mit der GPOH unter Berücksichtigung der weiteren Therapiemodalitäten, des Alters der Patienten und histologischer Kriterien abgestimmt. Bei der Indikationsstellung zur Radiotherapie ist die kurativ erforderliche Radikalität gegenüber möglichen chronischen Behandlungsfolgen abzuwägen.

Die Möglichkeiten der klassischen 3-Säulen-Therapie (Chirurgie, Chemotherapie und Strahlentherapie) erscheinen bei manchen Tumorerkrankungen nahezu ausgeschöpft zu sein, da die Heilungsraten bei den meisten kindlichen Krebserkrankungen trotz Intensivierung der Therapie stagnieren. Wesentliche Verbesserungen der Heilungsraten lassen sich mit einer Therapieintensivierung nicht mehr erreichen. Allerdings gelingt dank Einbindung der molekularen Diagnostik eine bessere Risikostratifizierung. Damit kann bei Niedrigrisikopatienten eine Überbehandlung mit dem möglichen Risiko langfristiger Therapienebenwirkungen vermieden werden. Insbesondere bei Hochrisikopatienten kamen auch in der pädiatrischen Onkologie zunehmend neue Therapiestrategien zur Anwendung. Molekular gezielte Medikamente und Immuntherapien werden bisher allerdings nahezu ausschließlich in der Rezidivsituation - und auch hier 
möglichst im Rahmen von Studien - erprobt.

\section{Herausforderung Jugendliche und junge Erwachsene (AYA)}

In der EU erkranken jährlich mehr als 12.000 Patienten im Alter von 15 bis 24 Jahren an Krebs. Adoleszente und junge Erwachsene fallen dabei oft in eine Kompetenzlücke zwischen Kinderund Erwachsenenonkologie und ihre altersspezifischen Belange und Bedürfnisse kommen häufig unzureichend zur Geltung. In den Kinderkliniken fehlt es oft an einer systematischen Entwicklung medizinischer und psychosozialer Angebote für Jugendliche. Die Vernetzung mit anderen Disziplinen bei der Betreuung jugendlicher Patienten mit Malignomen, die typischerweise ältere Patienten betreffen, stellt ebenso eine Herausforderung dar wie die Einbindung der pädiatrischen Onkologie bei jungen Erwachsenen mit typischen pädiatrischen Malignomen. Überregional nutzbare Strukturen zur Transition in die Erwachsenenmedizin existieren kaum. Diesen Themenfeldern widmet sich seit einigen Monaten eine neu gegründete multiprofessionelle Arbeitsgemeinschaft Adoleszente/junge Erwachsene/ Transition (AjET) der GPOH. Neben der Weiterentwicklung der Strukturen innerhalb der GPOH gilt das Augenmerk dabei der Vernetzung mit der Erwachsenen- und Organmedizin und internationalen Partnern sowie der Einbindung der Betroffenen.

In den folgenden Beiträgen möchten wir den Lesern einen komprimierten Überblick über die aktuelle Diagnostik und Therapie bei häufigen Krebserkrankungen des Kindes- und Jugendalters (Leukämien, Hirntumoren, Lymphome, embryonale Tumoren, Sarkome) und das wichtige Thema der Spätfolgen bieten.
Für die Schriftleiter

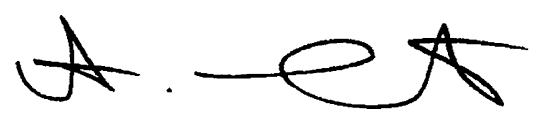

\section{A. Eggert}

Für die Herausgeber

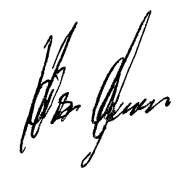

H. Schmidberger

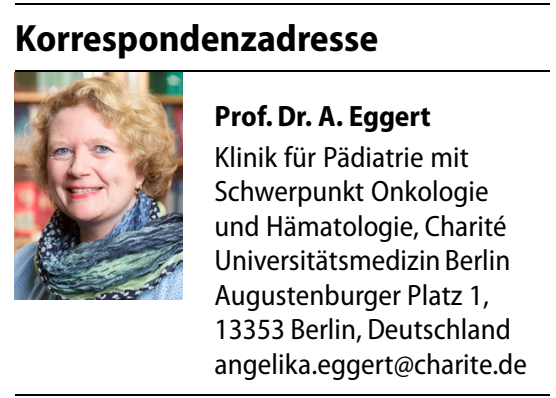

Interessenkonflikt. A. Eggert, D. Reinhardt, C. Kratz, U. Kontny, D.T. Schneider, H. Jürgens und H. Schmidberger geben an, dass kein Interessenkonflikt besteht. 